\title{
Association of Alcohol Consumption with the Incidence of Proteinuria and Chronic Kidney Disease: A Retrospective Cohort Study in Japan
}

\section{Akio Tanaka}

Aichi Medical University: Aichi Ika Daigaku

Makoto Yamaguchi

Aichi Medical University: Aichi lka Daigaku

Takuji Ishimoto

Aichi Medical University: Aichi Ika Daigaku

Takayuki Katsuno

Aichi Medical University: Aichi Ika Daigaku

Hironobu Nobata

Aichi Medical University: Aichi Ika Daigaku

Shiho Iwagaitsu

Aichi Medical University: Aichi Ika Daigaku

Hirokazu Sugiyama

Aichi Medical University: Aichi Ika Daigaku

Hiroshi Kinashi

Aichi Medical University: Aichi Ika Daigaku

\section{Shogo Banno}

Aichi Medical University: Aichi Ika Daigaku

Masahiko Ando

Nagoya University Hospital: Nagoya Daigaku Igakubu Fuzoku Byoin

\section{Yoko Kubo}

Nagoya University Graduate School of Medicine Faculty of Medicine: Nagoya Daigaku Daigakuin Igakukei Kenkyuka Igakubu

Yasuhiko Ito ( $\nabla$ yasuito@aichi-med-u.ac.jp )

Aichi Medical University: Aichi lka Daigaku https://orcid.org/0000-0002-9676-6961

\section{Research}

Keywords: alcohol, chronic kidney disease, proteinuria, sex difference, retrospective cohort study

Posted Date: October 21st, 2021 
DOI: https://doi.org/10.21203/rs.3.rs-965168/v1

License: (c) (1) This work is licensed under a Creative Commons Attribution 4.0 International License. Read Full License 


\section{Abstract \\ Background}

The difference in the clinical impact of alcohol consumption on kidney function based on sex remains to be elucidated. This study aimed to assess the association between the dose of alcohol consumption and the incidence of proteinuria and chronic kidney disease stratified by sex.

\section{Methods}

This retrospective cohort study included 26,788 workers (19,702 men and 7086 women) with normal renal function (estimated glomerular filtration rate $\geq 60 \mathrm{~mL} / \mathrm{min} / 1.73 \mathrm{~m}^{2}$ ) at annual health examinations between January 2010 and March 2015 in Japan. The main exposure was alcohol consumption. The primary outcomes were the incidence of proteinuria (dipstick urinary protein $\geq 1$ ) and incidence of chronic kidney disease (an estimated glomerular filtration rate $<60 \mathrm{~mL} / \mathrm{min}$ per $1.73 \mathrm{~m}^{2}$ and a $25 \%$ decrease from the baseline estimated glomerular filtration rate).

\section{Results}

During a median observational period of 4 years (interquartile range: 2-6), 1993 (10.1\%) men and 462 (6.5\%) women developed proteinuria, whereas 667 (3.4\%) men and 255 (3.6\%) women developed chronic kidney disease. After adjustment for clinically relevant factors using a Cox proportional hazards model, alcohol consumption of $\geq 40 \mathrm{~g} /$ day in females was significantly associated with the incidence of proteinuria (hazard ratio, 1.65; 95\% confidence interval, 1.09-2.51) and chronic kidney disease (hazard ratio, $1.77 ; 95 \%$ confidence interval, $1.09-2.85)$. However, no significant association between alcohol consumption and primary outcomes was observed in men.

\section{Conclusions}

In conclusion, daily higher alcohol consumption was significantly associated with a higher incidence of proteinuria and chronic kidney disease among women. Women might be prone to high alcohol consumption with kidney dysfunction.

\section{Background}

Chronic kidney disease (CKD) is a risk factor for cardiovascular disease, development of end-stage renal disease (ESRD), and all-cause mortality. Furthermore, CKD is considered an important global health problem owing to its socioeconomic burden [1]. Therefore, it is essential to identify the modifiable risk factors for the development of CKD and effective strategies for preventing it. 
Alcohol consumption is a lifestyle-related factor associated with mortality. Although excess alcohol intake has been associated with increased mortality [2], moderate drinkers generally have lower mortality rates than non-drinkers or heavy drinkers in population-based observational studies, namely demonstrating J-shaped associations [3-7]. As for the influence of alcohol on kidney function, although several population-based cohort studies have evaluated the relationship between the amount of alcohol consumption and incidence of CKD, these results have been inconsistent; an inverse association [8-11], a positive association [12-15], and a J-shaped association [16-21] between alcohol intake and incidence of CKD have been reported in longitudinal studies including the general population. Furthermore, although several studies have evaluated the difference in the clinical impact of alcohol consumption on kidney function based on sex $[10,15,20,21]$, the results have been inconsistent.

This retrospective cohort study of 26,788 individuals from the general population in Japan aimed to assess the association between the dose of alcohol consumption and the incidence of proteinuria and CKD stratified by sex. The results of this study may provide important implications for the clinical effect of alcohol consumption as a key lifestyle-related factor associated with the incidence of CKD.

\section{Methods}

\section{Study population}

A total of 42,833 participants from Daido Hospital aged $\geq 18$ years were included in the study. They received annual health checkups between January 2010 and March 2015 (baseline period). Of 38,521 (89.9\%) participants aged $\geq 20$ years with no proteinuria (dipstick urinary protein $\leq \pm$ ) and an estimated glomerular filtration rate (eGFR) $\geq 60 \mathrm{~mL} / \mathrm{min} / 1.73 \mathrm{~m}^{2}, 589$ with missing data on alcohol consumption, and 10,929 without two eGFR measurements with a 1-year interval during the observational period were excluded. Finally, 26,788 participants (19,702 men and 7086 women) with normal renal function (no proteinuria and eGFR $\geq 60 \mathrm{~mL} / \mathrm{min} / 1.73 \mathrm{~m}^{2}$ ) were included for analysis (Fig. 1).

To calculate eGFR, the following Japanese equation was used: eGFR $\left(\mathrm{mL} / \mathrm{min} / 1.73 \mathrm{~m}^{2}\right)=194 \times$ age (years) $)^{-0.287} \times$ serum creatinine $(\mathrm{mg} / \mathrm{dL})^{-1.094} \times 0.739$, if female [22].

The protocol of the present study was approved by the Daido Hospital and Aichi Medical University (no. 2020-8). An opt-out approach was adopted regarding informed patient consent, according to the Japanese Ethical Guidelines for Medical and Health Research involving human subjects.

\section{Measurements}

\section{Predictors and covariates}

The baseline clinical characteristics included age, sex, body mass index (BMI), systolic and diastolic blood pressure, serum levels of total cholesterol, triglyceride, hemoglobin A1c (NGSP), eGFR, and urinary 
protein level in the random void urine sample assessed by the dipstick method: negative $(-), \pm, 1+, 2+$, or $3+$.

Information about the lifestyle (alcohol consumption and smoking status [non-smoker, past smoker, and current smoker]) and current treatment for comorbidities at baseline (hypertension, hyperlipidemia, diabetes mellitus, and cardiovascular disease) were collected from self-administered questionnaires. The main exposure of the present study was the amount of alcohol consumed; in Japan, as a standard serving size of an alcoholic beverage, " $1 \mathrm{go}$ " is equivalent to approximately $180 \mathrm{~mL}$ of Japanese sake, or $23 \mathrm{~g}$ of ethanol, and is the most common unit for measuring the amount of alcohol consumed. Information was collected on daily alcohol consumption and frequency per week, and the average daily alcohol intake was categorized as no consumption, $<10 \mathrm{~g} /$ day, $10-19.9 \mathrm{~g} /$ day, $20-39.9 \mathrm{~g} /$ day, and $\geq 40$ $\mathrm{g} /$ day, according to a previous study [21].

\section{Outcomes}

The primary outcomes of interest were 1 ) incident proteinuria, defined as a urinary protein $\geq 1+$, as assessed by the dipstick test and 2) incident CKD, defined as two eGFR levels $<60 \mathrm{~mL} / \mathrm{min} / 1.73 \mathrm{~m}^{2}$ and a $25 \%$ decrease from baseline eGFR [23] available between the cohort entry date and December 1, 2018 (the last date of available eGFR measurements).

\section{Statistical analysis}

Baseline characteristics according to alcohol consumption categories in men and women were compared using the Kruskal-Wallis test or Pearson's chi-square test, as appropriate. Patients were followed up until the last date of the medical check-up. The associations of alcohol consumption with outcomes were assessed using the Kaplan-Meier method, log-rank tests, and Cox proportional hazards models (for timeto-event analyses).

Multivariate models were incrementally adjusted for the following clinically relevant factors: model 1 was adjusted for age (year), BMI $\left(\mathrm{kg} / \mathrm{m}^{2}\right)$, smoking status (never/former and current smokers); model 2 additionally included the eGFR $\left(\mathrm{mL} / \mathrm{min} / 1.73 \mathrm{~m}^{2}\right)$ at baseline and current treatment for comorbidities (hypertension, dyslipidemia, diabetes mellitus, and cardiovascular disease); and model 3 additionally accounted for systolic blood pressure $(\mathrm{mmHg})$ and the $\mathrm{HbA1c}(\%)$, total cholesterol (mg/dL), and triglyceride $(\mathrm{mg} / \mathrm{dL}$ ) levels. The proportionality assumption was tested by plotting log [2log (survival rate)] against log (survival time). For the time-to-event analyses, patients were followed up until censored at the date of the last check-up or December 13,2018 , for incident proteinuria and CKD, respectively. All associations were examined using unadjusted and multivariable-adjusted models. All analyses were performed separately in males and females. Continuous variables were expressed as medians and interquartile ranges and categorical variables as numbers and proportions. Statistical significance was set at $P<.05$. Statistical analyses were performed using Stata software version 15.0 (StataCorp LP, College Station, TX, USA) and JMP software version 14.0.0 (SAS Institute, Cary, NC, USA). 


\section{Results \\ Baseline characteristics}

The baseline characteristics of 19,902 men and 7086 women according to alcohol consumption categories are listed in Tables 1 and 2. 
Table 1

Baseline characteristics of 19,902 males

\begin{tabular}{|c|c|c|c|c|c|c|}
\hline & \multicolumn{6}{|c|}{ Daily alcohol consumption categories (g of alcohol) } \\
\hline & No & $<10 \mathrm{~g}$ & $10-19.9$ & 20-39.9 & $\geq 40$ & $\begin{array}{l}\mathrm{P}- \\
\text { value }\end{array}$ \\
\hline Number & 7352 & 4444 & 3226 & 2919 & 1761 & \\
\hline Age (years) & $\begin{array}{l}39(31- \\
49)\end{array}$ & $\begin{array}{l}39(31- \\
48)\end{array}$ & $\begin{array}{l}44(35- \\
55)\end{array}$ & $48(40-57)$ & $48(40-57)$ & $<.001$ \\
\hline Smoking, n (\%) & & & & & & $<.001$ \\
\hline Never & $\begin{array}{l}2793 \\
(38.0)\end{array}$ & $\begin{array}{l}1629 \\
(36.7)\end{array}$ & $762(23.6)$ & $475(16.3)$ & $195(11.1)$ & \\
\hline Former & $\begin{array}{l}1714 \\
(23.3)\end{array}$ & $\begin{array}{l}1233 \\
(27.8)\end{array}$ & $\begin{array}{l}1137 \\
(35.2)\end{array}$ & $\begin{array}{l}1072 \\
(36.7)\end{array}$ & $647(36.7)$ & \\
\hline Current & $\begin{array}{l}2803 \\
(38.1)\end{array}$ & $\begin{array}{l}1582 \\
(35.6)\end{array}$ & $\begin{array}{l}1327 \\
(41.1)\end{array}$ & $\begin{array}{l}1372 \\
(47.0)\end{array}$ & $919(52.2)$ & \\
\hline $\begin{array}{l}\text { Body mass index, } \\
\mathrm{kg} / \mathrm{m}^{2}\end{array}$ & $\begin{array}{l}23.3 \\
(21.1- \\
25.9)\end{array}$ & $\begin{array}{l}23.1 \\
(21.2- \\
25.2)\end{array}$ & $\begin{array}{l}23.4 \\
(21.6- \\
25.6)\end{array}$ & $\begin{array}{l}23.2 \\
(21.5- \\
25.1)\end{array}$ & $\begin{array}{l}23.3 \\
(21.5- \\
25.3)\end{array}$ & $<.001$ \\
\hline Systolic BP, mmHg & $\begin{array}{l}119(109- \\
130)\end{array}$ & $\begin{array}{l}119(110- \\
129)\end{array}$ & $\begin{array}{l}120(110- \\
132)\end{array}$ & $\begin{array}{l}121(111- \\
133)\end{array}$ & $\begin{array}{l}121(111- \\
132)\end{array}$ & $<.001$ \\
\hline $\begin{array}{l}\text { Diastolic BP, } \\
\mathrm{mmHg}\end{array}$ & $\begin{array}{l}72(65- \\
81)\end{array}$ & $\begin{array}{l}73(65- \\
81)\end{array}$ & $\begin{array}{l}74(66- \\
83)\end{array}$ & $75(67-84)$ & $76(67-84)$ & $<.001$ \\
\hline Uric acid, $\mathrm{mg} / \mathrm{dL}$ & $\begin{array}{l}5.8(4.9- \\
6.7)\end{array}$ & $\begin{array}{l}5.8(5.0- \\
6.7)\end{array}$ & $\begin{array}{l}5.8(5.0- \\
6.7)\end{array}$ & $\begin{array}{l}5.9(5.0- \\
6.7)\end{array}$ & $\begin{array}{l}5.9(5.0- \\
6.7)\end{array}$ & .341 \\
\hline $\begin{array}{l}\text { Total cholesterol, } \\
\mathrm{mg} / \mathrm{dL}\end{array}$ & $\begin{array}{l}196(173- \\
222)\end{array}$ & $\begin{array}{l}196(174- \\
220)\end{array}$ & $\begin{array}{l}201(179- \\
225)\end{array}$ & $\begin{array}{l}203(183- \\
226)\end{array}$ & $\begin{array}{l}205(183- \\
227)\end{array}$ & $<.001$ \\
\hline Triglyceride, mg/dL & $\begin{array}{l}96(66- \\
142)\end{array}$ & $\begin{array}{l}95(65- \\
142)\end{array}$ & $\begin{array}{l}99(69- \\
145)\end{array}$ & $\begin{array}{l}103(72- \\
152)\end{array}$ & $\begin{array}{l}109(74- \\
171)\end{array}$ & $<.001$ \\
\hline Hemoglobin A1c, \% & $\begin{array}{l}5.4(5.2- \\
5.7)\end{array}$ & $\begin{array}{l}5.4(5.2- \\
5.6)\end{array}$ & $\begin{array}{l}5.5(5.2- \\
5.7)\end{array}$ & $\begin{array}{l}5.5(5.2- \\
5.7)\end{array}$ & $\begin{array}{l}5.4(5.2- \\
5.7)\end{array}$ & $<.001$ \\
\hline $\begin{array}{l}\text { eGFR, } \\
\mathrm{mL} / \mathrm{min} / 1.73 \mathrm{~m}^{2}\end{array}$ & $\begin{array}{l}87(77- \\
97)\end{array}$ & $\begin{array}{l}86(77- \\
96)\end{array}$ & $\begin{array}{l}84(75- \\
94)\end{array}$ & $83(74-93)$ & $85(76-96)$ & $<.001$ \\
\hline \multicolumn{7}{|l|}{ Current treatments } \\
\hline Hypertension, n (\%) & $426(5.8)$ & $275(6.2)$ & $406(12.6)$ & $430(14.7)$ & $306(17.4)$ & $<.001$ \\
\hline Dyslipidemia, n (\%) & $242(3.3)$ & $171(3.9)$ & $190(5.9)$ & $106(3.6)$ & $84(4.8)$ & $<.001$ \\
\hline $\begin{array}{l}\text { Diabetes mellitus, } \\
\mathrm{n}(\%)\end{array}$ & $210(2.9)$ & $108(2.4)$ & 115 (3.6) & $99(3.4)$ & $69(3.9)$ & .004 \\
\hline
\end{tabular}




\begin{tabular}{|lllllll|}
\hline \multicolumn{7}{|c|}{ Daily alcohol consumption categories (g of alcohol) } \\
\hline CVD, n (\%) & $37(0.5)$ & $12(0.3)$ & $27(0.8)$ & $21(0.7)$ & $8(0.5)$ & .009 \\
\hline $\begin{array}{l}\text { Observational } \\
\text { period (years) }\end{array}$ & $4(2-6)$ & $4(3-6)$ & $5(3-6)$ & $5(3-6)$ & $5(2-6)$ & $<.001$ \\
\hline
\end{tabular}


Table 2

Baseline characteristics of 7086 females

\section{Daily alcohol consumption categories ( $\mathrm{g}$ of alcohol)}

\begin{tabular}{|c|c|c|c|c|c|c|}
\hline & No & $<10 \mathrm{~g}$ & $10-19.9$ & $20-39.9$ & $\geq 40$ & $\begin{array}{l}\mathrm{P} \text { - } \\
\text { value }\end{array}$ \\
\hline Number & 4017 & 1359 & 724 & 611 & 375 & \\
\hline Age (years) & $\begin{array}{l}42(35- \\
51)\end{array}$ & $\begin{array}{l}40(34- \\
48)\end{array}$ & $\begin{array}{l}46(39- \\
55)\end{array}$ & $46(40-56)$ & $47(39-55)$ & $<.001$ \\
\hline Smoking, n (\%) & & & & & & $<.001$ \\
\hline Never & $\begin{array}{l}3258 \\
(81.1)\end{array}$ & $\begin{array}{l}1076 \\
(79.2)\end{array}$ & $497(68.7)$ & $405(66.3)$ & $247(65.9)$ & \\
\hline Former & $404(10.1)$ & $184(13.5)$ & $122(16.9)$ & $104(17.0)$ & $68(18.1)$ & \\
\hline Current & $301(7.5)$ & $99(7.3)$ & $101(14.0)$ & $96(15.7)$ & $57(15.2)$ & \\
\hline $\begin{array}{l}\text { Body mass index, } \\
\mathrm{kg} / \mathrm{m}^{2}\end{array}$ & $\begin{array}{l}21.1 \\
(19.3- \\
23.6)\end{array}$ & $\begin{array}{l}20.9 \\
(19.2- \\
22.9)\end{array}$ & $\begin{array}{l}21.5 \\
(19.6- \\
24.1)\end{array}$ & $\begin{array}{l}21.6 \\
(19.7- \\
24.3)\end{array}$ & $\begin{array}{l}21.8 \\
(19.8- \\
24.2)\end{array}$ & $<.001$ \\
\hline Systolic BP, mmHg & $\begin{array}{l}112(102- \\
125)\end{array}$ & $\begin{array}{l}112(102- \\
124)\end{array}$ & $\begin{array}{l}111(103- \\
124)\end{array}$ & $\begin{array}{l}112(102- \\
125)\end{array}$ & $\begin{array}{l}115(104- \\
126)\end{array}$ & .649 \\
\hline $\begin{array}{l}\text { Diastolic BP, } \\
\text { mmHg }\end{array}$ & $\begin{array}{l}68(61- \\
76)\end{array}$ & $\begin{array}{l}68(61- \\
76)\end{array}$ & $\begin{array}{l}68(61- \\
76)\end{array}$ & $68(61-77)$ & $70(61-77)$ & .408 \\
\hline Uric acid, mg/dL & $\begin{array}{l}4.5(3.8- \\
5.5)\end{array}$ & $\begin{array}{l}4.6(3.9- \\
5.5)\end{array}$ & $\begin{array}{l}4.5(3.8- \\
5.4)\end{array}$ & $\begin{array}{l}4.5(3.7- \\
5.4)\end{array}$ & $\begin{array}{l}4.5(3.8- \\
5.4)\end{array}$ & .315 \\
\hline $\begin{array}{l}\text { Total cholesterol, } \\
\mathrm{mg} / \mathrm{dL}\end{array}$ & $\begin{array}{l}199(178- \\
225)\end{array}$ & $\begin{array}{l}196(174- \\
221)\end{array}$ & $\begin{array}{l}204(181- \\
227)\end{array}$ & $\begin{array}{l}203(180- \\
229)\end{array}$ & $\begin{array}{l}202(180- \\
230)\end{array}$ & $<.001$ \\
\hline Triglyceride, mg/dL & $\begin{array}{l}70(51- \\
98)\end{array}$ & $\begin{array}{l}65(50- \\
90)\end{array}$ & $\begin{array}{l}75(55- \\
108)\end{array}$ & $\begin{array}{l}76(59- \\
107)\end{array}$ & $\begin{array}{l}84(61- \\
126)\end{array}$ & $<.001$ \\
\hline Hemoglobin A1c, \% & $\begin{array}{l}5.4(5.2- \\
5.7)\end{array}$ & $\begin{array}{l}5.4(5.2- \\
5.6)\end{array}$ & $\begin{array}{l}5.4(5.2- \\
5.7)\end{array}$ & $\begin{array}{l}5.4(5.2- \\
5.7)\end{array}$ & $\begin{array}{l}5.4(5.2- \\
5.7)\end{array}$ & $<.001$ \\
\hline $\begin{array}{l}\text { eGFR, } \\
\mathrm{mL} / \mathrm{min} / 1.73 \mathrm{~m}^{2}\end{array}$ & $\begin{array}{l}75(68- \\
84)\end{array}$ & $\begin{array}{l}76(68- \\
85)\end{array}$ & $\begin{array}{l}75(67- \\
83)^{-}\end{array}$ & $73(67-82)$ & $76(68-83)$ & $<.001$ \\
\hline \multicolumn{7}{|l|}{ Current treatments } \\
\hline Hypertension, n (\%) & $188(4.7)$ & $42(3.1)$ & $63(8.7)$ & $65(10.6)$ & $44(11.7)$ & $<.001$ \\
\hline Dyslipidemia, n (\%) & $147(3.7)$ & $34(2.5)$ & $34(4.7)$ & $38(6.2)$ & $26(6.9)$ & $<.001$ \\
\hline $\begin{array}{l}\text { Diabetes mellitus, } \\
\text { n (\%) }\end{array}$ & $50(1.2)$ & $8(0.6)$ & $13(1.8)$ & $10(1.6)$ & $8(2.1)$ & .047 \\
\hline CVD, n (\%) & $11(0.3)$ & $1(0.1)$ & $2(0.3)$ & $3(0.5)$ & $1(0.3)$ & .528 \\
\hline
\end{tabular}




\begin{tabular}{|lllllll|}
\hline \multicolumn{7}{|c|}{ Daily alcohol consumption categories (g of alcohol) } \\
\hline $\begin{array}{l}\text { Observational } \\
\text { period (years) }\end{array}$ & $4(2-5)$ & $3(2-5)$ & $4(2-5)$ & $4(2-6)$ & $4(2-6)$ & $<.001$ \\
\hline
\end{tabular}

Among male participants, non-drinkers $[n=7352$ (37.3\%)] were the most common, followed by those with consumption levels of $<10 \mathrm{~g} /$ day $[n=4444(22.6 \%)], 10-19.9 \mathrm{~g} /$ day $[n=3226(16.4 \%)], 20-39.9[n=$ 2919 (14.8\%)], and $\geq 40 \mathrm{~g} /$ day $[n=1761$ (8.9\%)] (Table 1). Individuals with a higher alcohol consumption tended to be older, smokers, dyslipidemic, receiving treatment for hypertension, and diabetic.

Among women, non-drinkers were the most common $[n=4017(56.7 \%)]$, which was at a higher proportion than that in men, followed by those consuming $<10 \mathrm{~g} /$ day $[n=1359(19.2 \%)], 10-19.9 \mathrm{~g} /$ day $[n=724$ (10.2\%)], 20-39.9 g/ day $[n=611(8.6 \%)]$, and $\geq 40 \mathrm{~g} /$ day $[n=375$ (5.3\%)] (Table 2). Similar trends were observed for age, smoking, dyslipidemia, and treatment for hypertension.

\section{Amount of alcohol consumption and risk of incident proteinuria}

During an observational period of 4 (3-6) years, $1993(10.1 \%)$ males developed proteinuria $(\geq 1+)($ Table 3). With respect to frequency of alcohol consumption, the incidence rates of proteinuria were 26.7, 24.3, $26.8,23.8$, and 29.8 per 1000 person-years in the "no," "<10 g," "10-19.9 g," "20-39.9 g," and " $\geq 40 \mathrm{~g}$ " consumption groups, respectively. The cumulative probability of incidence of proteinuria was comparable between the categories of alcohol consumption $(P=.057)$ (Fig. 2A). Both unadjusted and multivariate Cox proportional hazards models showed no significant association between alcohol consumption and the incidence of proteinuria (Table 3). 
Table 3

Alcohol consumption and the incidence of proteinuria in males and females

\section{Daily alcohol consumption categories ( $\mathrm{g}$ of alcohol)}

$\begin{array}{lllll}\text { No } & <10 & 10-19.9 & 20-39.9 & \geq 40 \quad \begin{array}{l}\text { P- } \\ \text { value }\end{array}\end{array}$

Males

\begin{tabular}{lllllll}
$\begin{array}{l}\text { Incidence of } \\
\text { proteinuria, } n(\%)\end{array}$ & $744(10.1)$ & $419(9.4)$ & $347(10.8)$ & $280(9.6)$ & $203(11.5)$ & .075 \\
\hline IR per 1000PY & 26.7 & 24.3 & 26.8 & 23.8 & 29.8
\end{tabular}

Hazard ratio $(95 \%$

$\mathrm{Cl})$

$\begin{array}{llllll}\text { Unadjusted model } & 1.0 & 0.91 & 0.99 & 0.88 & 1.10(0.94- \\ & \text { (reference) } & (0.80- & (0.87- & (0.76- & 1.28) \\ & & 1.02) & 1.13) & 1.00) & \end{array}$

$\begin{array}{llllll}\text { Adjusted model } 1 & 1.0 & 0.95 & 1.04 & 0.96 & 1.17(0.99- \\ & \text { (reference) } & (0.84- & (0.92- & (0.83- & 1.37) \\ & & 1.07) & 1.18) & 1.10) & \end{array}$

$\begin{array}{llllll}\text { Adjusted model } 2 & \begin{array}{l}1.0 \\ \text { (reference) }\end{array} & \begin{array}{l}0.95 \\ (0.85-\end{array} & \begin{array}{l}1.03 \\ (0.90-\end{array} & \begin{array}{l}0.95 \\ (0.82-\end{array} & \begin{array}{l}1.14(0.97- \\ 1.34)\end{array} \\ & & & 1.17) & 1.09) & \\ \text { Adjusted model } 3 & \begin{array}{l}1.0 \\ \text { (reference) }\end{array} & \begin{array}{llll}0.91 \\ (0.79-\end{array} & \begin{array}{l}1.04 \\ (0.89-\end{array} & \begin{array}{l}0.96 \\ (0.81-\end{array} & 1.13(0.92- \\ & & 1.05) & 1.21) & 1.13) & \end{array}$

\section{Females}

Incidence of

proteinuria, $n(\%)$

$257(6.4)$

$87(6.4)$

$44(6.1)$

$35(5.7)$

$39(10.4)$

.036

IR per 1000PY

18.0

18.8

16.8

15.1

28.1

Hazard ratio (95\%

$\mathrm{Cl})$

PY, person-years; $\mathrm{Cl}$, confidence interval.

Multivariate model 1 adjusted for age (years), body mass index (BMI) $\left(\mathrm{kg} / \mathrm{m}^{2}\right)$, smoking status (never/former and current smokers).

Model 2 adjusted for the covariates in model 1 , eGFR $\left(\mathrm{mL} / \mathrm{min} / 1.73 \mathrm{~m}^{2}\right)$ at baseline, and current treatment for comorbidities (hypertension, dyslipidemia, diabetes mellitus, and cardiovascular disease).

Model 3 adjusted for the covariates in model 2, systolic blood pressure $(\mathrm{mmHg}), \mathrm{HbA} 1 \mathrm{c}(\%)$, total cholesterol $(\mathrm{mg} / \mathrm{dL})$, and triglyceride $(\mathrm{mg} / \mathrm{dL})$.

$\star P<.05$. 


\begin{tabular}{|c|c|c|c|c|c|}
\hline \multicolumn{6}{|c|}{ Daily alcohol consumption categories ( $\mathrm{g}$ of alcohol) } \\
\hline Unadjusted model & $\begin{array}{l}1.0 \\
\text { (reference) }\end{array}$ & $\begin{array}{l}1.04 \\
(0.82- \\
1.33)\end{array}$ & $\begin{array}{l}0.94 \\
(0.68- \\
1.29)\end{array}$ & $\begin{array}{l}0.84 \\
(0.59- \\
1.20)\end{array}$ & $\begin{array}{l}1.56(1.11- \\
2.18)^{\star}\end{array}$ \\
\hline Adjusted model 1 & $\begin{array}{l}1.0 \\
\text { (reference) }\end{array}$ & $\begin{array}{l}1.00 \\
(0.79- \\
1.28)\end{array}$ & $\begin{array}{l}1.01 \\
(0.73- \\
1.39)\end{array}$ & $\begin{array}{l}0.94 \\
(0.65- \\
1.34)\end{array}$ & $\begin{array}{l}1.74(1.23- \\
2.45)^{\star}\end{array}$ \\
\hline Adjusted model 2 & $\begin{array}{l}1.0 \\
\text { (reference) }\end{array}$ & $\begin{array}{l}1.01 \\
(0.79- \\
1.29)\end{array}$ & $\begin{array}{l}1.00 \\
(0.72- \\
1.39)\end{array}$ & $\begin{array}{l}0.93 \\
(0.65- \\
1.34)\end{array}$ & $\begin{array}{l}1.72(1.21- \\
2.42)^{\star}\end{array}$ \\
\hline Adjusted model 3 & $\begin{array}{l}1.0 \\
\text { (reference) }\end{array}$ & $\begin{array}{l}1.03 \\
(0.78- \\
1.36)\end{array}$ & $\begin{array}{l}0.91 \\
(0.61- \\
1.35)\end{array}$ & $\begin{array}{l}1.04 \\
(0.69- \\
1.56)\end{array}$ & $1.65(1.09-$ \\
\hline \multicolumn{6}{|c|}{ PY, person-years; $\mathrm{Cl}$, confidence interval. } \\
\hline \multicolumn{6}{|c|}{$\begin{array}{l}\text { Multivariate model } 1 \text { adjusted for age (years), body mass index (BMI) }\left(\mathrm{kg} / \mathrm{m}^{2}\right) \text {, smoking status } \\
\text { (never/former and current smokers). }\end{array}$} \\
\hline \multicolumn{6}{|c|}{$\begin{array}{l}\text { Model } 2 \text { adjusted for the covariates in model } 1 \text {, eGFR }\left(\mathrm{mL} / \mathrm{min} / 1.73 \mathrm{~m}^{2}\right) \text { at baseline, and current } \\
\text { treatment for comorbidities (hypertension, dyslipidemia, diabetes mellitus, and cardiovascular } \\
\text { disease). }\end{array}$} \\
\hline \multicolumn{6}{|c|}{$\begin{array}{l}\text { Model } 3 \text { adjusted for the covariates in model } 2 \text {, systolic blood pressure }(\mathrm{mmHg}), \mathrm{HbA} 1 \mathrm{c}(\%) \text {, total } \\
\text { cholesterol }(\mathrm{mg} / \mathrm{dL}) \text {, and triglyceride }(\mathrm{mg} / \mathrm{dL}) \text {. }\end{array}$} \\
\hline$\star P<.05$. & & & & & \\
\hline
\end{tabular}

Among women, during the observational period of $4(2-5)$ years, $462(6.5 \%)$ developed proteinuria $(\geq 1+)$ (Table 3). With respect to frequency of alcohol consumption, the incidence rates of proteinuria were 18.0, $18.8,16.8,15.1$, and 28.1 per 1000 person-years in the "no," "<10 g," "10-19.9 g," "20-39.9 g," and " $\geq 40$ $\mathrm{g}$ " consumption groups, respectively. The cumulative incidence of proteinuria in females was significantly higher in the $>40 \mathrm{~g}$ /day alcohol consumption group (log rank, $P=.049$ ) (Fig. 2B). Unadjusted Cox proportional hazards models showed that $\geq 40 \mathrm{~g}$ alcohol consumption was significantly associated with the incidence of proteinuria [hazard ratios (95\% confidence interval): non-drinker, 1.00 (reference), $<10 \mathrm{~g}$, 1.04 (0.82-1.33), 10-19.9 g, 0.94 (0.68-1.29), 20-39.9 g, 0.84 (0.59-1.20), and $\geq 40 \mathrm{~g}, 1.56$ (1.11-2.18), respectively] (Table 3). After adjusting for clinically relevant factors, $\geq 40 \mathrm{~g}$ consumption was identified as a significant predictor of the incidence of proteinuria [non-drinker, 1.00 (reference), <10 g, 1.03 (0.781.36), 10-19.9 g, 0.91 (0.61-1.35), 20-39.9 g, 1.04 (0.69-1.56), and $\geq 40 \mathrm{~g}, 1.65$ (1.09-2.51), respectively] (model 3, Table 3).

\section{Amount of alcohol consumption and risk of incident CKD}

Among men, a total of 719 (3.5\%) developed CKD during the observation period (Table 4). A higher incidence rate of CKD was observed in men in $\geq 40 \mathrm{~g}$ drinkers (Table 4). With respect to categories of 
alcohol consumption, the incidence rates of CKD were 8.4, 8.1, 8.4, 8.8, and 13.0 per 1000 person-years in the "no," "<10 g," "10-19.9 g," "20-39.9 g," and " $\geq 40$ g" groups, respectively. The cumulative incidence of CKD was slightly higher in males in the " $\geq 40 \mathrm{~g}$ " groups ( $P=.016)$ (Fig. 2C). Although unadjusted Cox proportional hazards models showed that $\geq 40 \mathrm{~g}$ of alcohol consumption was associated with the incidence of CKD, multivariate models showed that the incidence of CKD was comparable among males between different alcohol consumption groups (Table 4). 
Table 4

Alcohol consumption and the incidence of CKD in males and females

Daily alcohol consumption categories ( $\mathrm{g}$ of alcohol)

No $\quad<10 \mathrm{~g} \quad 10-19.9 \quad 20-39.9 \quad \geq 40 \quad \begin{aligned} & \mathrm{P} \\ & \text { value }\end{aligned}$

\section{Males}

$\begin{array}{lcccccc}\begin{array}{l}\text { Incidence of } \\ \text { CKD, } n(\%)\end{array} & 228(3.1) & 143(3.2) & 108(3.4) & 105(3.6) & 83(4.7) & .017 \\ \text { IR per 1000PY } & 8.4 & 8.1 & 8.4 & 8.8 & 13.0\end{array}$

Hazard ratio

$(95 \% \mathrm{Cl})$

\begin{tabular}{|c|c|c|c|c|c|}
\hline $\begin{array}{l}\text { Unadjusted } \\
\text { model }\end{array}$ & $\begin{array}{l}1.0 \\
\text { (reference) }\end{array}$ & $\begin{array}{l}1.02 \\
(0.82- \\
1.25)\end{array}$ & $\begin{array}{l}1.00(0.80- \\
1.26)\end{array}$ & $\begin{array}{l}1.00(0.80- \\
1.26)\end{array}$ & $\begin{array}{l}1.32(1.03- \\
1.70)^{\star}\end{array}$ \\
\hline $\begin{array}{l}\text { Adjusted model } \\
1\end{array}$ & $\begin{array}{l}1.0 \\
\text { (reference) }\end{array}$ & $\begin{array}{l}1.06 \\
(0.86- \\
1.31)\end{array}$ & $\begin{array}{l}0.91(0.72- \\
1.14)\end{array}$ & $\begin{array}{l}0.95(0.75- \\
1.20)\end{array}$ & $\begin{array}{l}1.21(0.94- \\
1.57)\end{array}$ \\
\hline
\end{tabular}

$\begin{array}{llllll}\text { Adjusted model } & 1.0 & 1.07 & 0.90(0.72- & 0.91(0.72- & 0.92(0.71- \\ 2 & \text { (reference) } & (0.87- & 1.14) & 1.16) & 1.20) \\ & & 1.33) & & & \end{array}$

$\begin{array}{llllll}\text { Adjusted model } & 1.0 & 1.17 & 0.90(0.68- & 0.93(0.71- & 0.95(0.69- \\ 3 & \text { (reference) } & (0.92- & 1.17) & 1.23) & 1.30) \\ 1.49) & & & & \end{array}$

\section{Females}

$\begin{array}{lcccccc}\begin{array}{l}\text { Incidence of } \\ \text { CKD, } n(\%)\end{array} & 120(3.0) & 43(3.2) & 27(3.7) & 35(5.7) & 30(8.0) & <.001 \\ \text { IR per 1000PY } & 8.3 & 9.0 & 10.2 & 15.5 & 20.9\end{array}$

Hazard ratio

$(95 \% \mathrm{Cl})$

PY, person-years; $\mathrm{Cl}$, confidence interval.

Multivariate model 1 adjusted for age (years), body mass index (BMI) $\left(\mathrm{kg} / \mathrm{m}^{2}\right)$, smoking status (never/former and current smokers).

Model 2 adjusted for the covariates in model 1, eGFR $\left(\mathrm{mL} / \mathrm{min} / 1.73 \mathrm{~m}^{2}\right)$ at baseline, and current treatment for comorbidities (hypertension, dyslipidemia, diabetes mellitus, and cardiovascular disease).

Model 3 adjusted for the covariates in model 2, systolic blood pressure $(\mathrm{mmHg}), \mathrm{HbA} 1 \mathrm{c}(\%)$, total cholesterol $(\mathrm{mg} / \mathrm{dL})$, and triglyceride $(\mathrm{mg} / \mathrm{dL})$.

$\star P<.05$ 


\section{Daily alcohol consumption categories (g of alcohol)}

\begin{tabular}{|c|c|c|c|c|c|}
\hline $\begin{array}{l}\text { Unadjusted } \\
\text { model }\end{array}$ & $\begin{array}{l}1.0 \\
\text { (reference) }\end{array}$ & $\begin{array}{l}1.12 \\
(0.79- \\
1.59)\end{array}$ & $\begin{array}{l}1.20(0.79- \\
1.82)\end{array}$ & $\begin{array}{l}1.61(1.11- \\
2.35) \star\end{array}$ & $\begin{array}{l}2.32(1.55- \\
3.46) *\end{array}$ \\
\hline $\begin{array}{l}\text { Adjusted model } \\
1\end{array}$ & $\begin{array}{l}1.0 \\
\text { (reference) }\end{array}$ & $\begin{array}{l}1.10 \\
(0.78- \\
1.56)\end{array}$ & $\begin{array}{l}1.30(0.85- \\
1.98)\end{array}$ & $\begin{array}{l}1.75(1.19- \\
2.57) \star\end{array}$ & $\begin{array}{l}2.66(1.77- \\
3.99) *\end{array}$ \\
\hline $\begin{array}{l}\text { Adjusted model } \\
2\end{array}$ & $\begin{array}{l}1.0 \\
\text { (reference) }\end{array}$ & $\begin{array}{l}1.09 \\
(0.77- \\
1.55)\end{array}$ & $\begin{array}{l}1.29(0.85- \\
1.97)\end{array}$ & $\begin{array}{l}1.33(0.89- \\
1.99)\end{array}$ & $\begin{array}{l}1.72(1.12- \\
2.64) *\end{array}$ \\
\hline $\begin{array}{l}\text { Adjusted model } \\
3\end{array}$ & $\begin{array}{l}1.0 \\
\text { (reference) }\end{array}$ & $\begin{array}{l}1.18 \\
(0.80- \\
1.74)\end{array}$ & $\begin{array}{l}1.21(0.74- \\
1.98)\end{array}$ & $\begin{array}{l}1.27(0.80- \\
2.02)\end{array}$ & $\begin{array}{l}1.77(1.09- \\
2.85) *\end{array}$ \\
\hline
\end{tabular}

PY, person-years; $\mathrm{Cl}$, confidence interval.

Multivariate model 1 adjusted for age (years), body mass index (BMI) $\left(\mathrm{kg} / \mathrm{m}^{2}\right)$, smoking status (never/former and current smokers).

Model 2 adjusted for the covariates in model 1, eGFR $\left(\mathrm{mL} / \mathrm{min} / 1.73 \mathrm{~m}^{2}\right)$ at baseline, and current treatment for comorbidities (hypertension, dyslipidemia, diabetes mellitus, and cardiovascular disease).

Model 3 adjusted for the covariates in model 2, systolic blood pressure (mmHg), HbA1c (\%), total cholesterol $(\mathrm{mg} / \mathrm{dL})$, and triglyceride $(\mathrm{mg} / \mathrm{dL})$.

$\star P<.05$.

Among women, the incidence of CKD was observed in 264 (3.6\%) during the observational period (Table 4). In contrast to men, the incidence rates of CKD were significantly higher in the $\geq 40 \mathrm{~g}$ group (Table 4); the incidence rates of CKD were 8.3, 9.0, 10.2, 15.5, and 20.9 per 1000 person-years in the " $<19 \mathrm{~g} /$ day," "19-30 g/day" and " $\geq 40 \mathrm{~g}$ " groups, respectively. The cumulative probabilities of the incidence of CKD were significantly higher in the $\geq 40 \mathrm{~g}$ group $(P<.001)$ (Fig. 2D).

Unadjusted Cox proportional hazards models showed that $\geq 40 \mathrm{~g}$ of alcohol consumption was significantly associated with the incidence of CKD [hazard ratios ( $95 \%$ confidence interval); non-drinker, 1.00 (reference); <10 g, 1.12 (0.79-1.59); 10-19.9 g, 1.20 (0.79-1.82); 20-39.9 g, 1.61 (1.11-2.35); and $\geq 40 \mathrm{~g}, 2.32$ (1.55-3.46), respectively] (Table 4). After adjusting for clinically relevant factors, $\geq 40 \mathrm{~g}$ consumption was also identified as a significant predictor of the incidence of CKD [non-drinker, 1.00 (reference); <10 g, 1.18 (0.80-1.74); 10-19.9 g, 1.21 (0.74-1.98); 20-39.9 g, 1.27 (0.80-2.02); and $\geq 40$ g, 1.77 (1.09-2.85), respectively] (model 3, Table 4).

\section{Discussion}


The present cohort study, including 26,788 members of the general population with non-CKD, revealed that a daily higher alcohol consumption ( $\geq 40 \mathrm{~g} /$ day) was significantly associated with a higher incidence of proteinuria and CKD among women. These findings suggest that women might be vulnerable to excess alcohol consumption, which might lead to kidney dysfunction. An advantage of the present study confirmed the robustness of the influence of alcohol consumption on two clinically important outcome measures of kidney dysfunction (incidence of proteinuria and eGFR $<60 \mathrm{~mL} / \mathrm{min} / 1.73 \mathrm{~m}^{2}$ with $25 \%$ decline).

Although multiple observational cohort studies have evaluated the relationship between alcohol consumption and incidence of CKD [24], incidence of proteinuria [9, 15, 21, 25], incidence of low eGFR [8, $9,12,16]$, and GFR annual decline [20], these results were not entirely consistent, possibly due to different sample sizes, different definitions of alcohol intake levels and CKD outcome measures, or different lifestyles of participants.

Among several large cohort studies that evaluated the relationship between alcohol consumption and incidence of proteinuria, Yamagata et al. reported that an average daily alcohol consumption of $\leq 20 \mathrm{~g}$ of ethanol was associated with a decreased risk of future proteinuria in Japanese men and women compared with non-drinkers, while consumption of $>20 \mathrm{~g}$ of ethanol was not associated with the risk of future proteinuria [21]. However, this study did not evaluate the clinical impact of heavy alcohol consumption on the incidence of proteinuria. Uehara et al. showed a significant influence of high alcohol consumption ( $>69 \mathrm{~g} /$ day) [26], which showed a J-shaped association in 9154 non-diabetic Japanese men. However, this study did not include women, and thus, its influence on women was unknown. Recently, Kimura et al. showed that women were more vulnerable to high doses of alcohol consumption ( $\geq 60 \mathrm{~g} /$ day) than men for the incidence of proteinuria in a general population-based large retrospective cohort study, including 11,286 Japanese male and female participants [15]. Although it was compatible with the present study, this study only focused on the incidence of proteinuria as a surrogate marker of end-stage renal disease (ESRD); it should be evaluated by other clinically important surrogate renal outcomes, such as eGFR.

Meanwhile, among the other few studies that evaluated not only the incidence of proteinuria but also eGFR decline as a CKD surrogate marker, one prospective cohort study including 6259 Australian male and female participants showed that the risk of developing new-onset albuminuria began to rise for an average daily alcohol consumption ( $\geq 30 \mathrm{~g} /$ day), compared with consumption of $<10 \mathrm{~g} /$ day [9]. However, inversely, the risk of developing a low eGFR was significantly reduced at the same threshold. This discrepancy should be cautiously interpreted. In addition, another recent large, nationwide, retrospective cohort study in Korea, including 118,492 participants, assessed the relationship between alcohol consumption and the incidence of CKD, defined as the incidence of proteinuria, low eGFR (eGFR $<60$ $\mathrm{mL} / \mathrm{min} / 1.73 \mathrm{~m}^{2}$ ), and eGFR annual decline [20]. It showed that a J-shaped association of alcohol consumption with incident proteinuria was observed in men; a lower risk in men drinking $<10 \mathrm{~g}$ of alcohol/day and a higher risk in men drinking higher levels of alcohol ( $\geq 40 \mathrm{~g} /$ day) were observed compared to those in non-drinkers, and a positive association was seen in women who consumed 
alcohol with any range of consumption compared with non-drinkers. However, when evaluating the low eGFR incidence and eGFR annual decline as outcomes, a negative association between alcohol intake and outcomes was observed in both sexes. These results were conflicting in each CKD outcome definition; therefore, these results should be interpreted cautiously. Meanwhile, in another large, prospective, population-based cohort, including 5476 male and female participants in the Netherlands, alcohol consumption was inversely associated with the risk of developing CKD, defined as either an eGFR $<60 \mathrm{~mL} / \mathrm{min} / 1.73 \mathrm{~m}^{2}$ or proteinuria (24-hour urinary albumin excretion $>30 \mathrm{mg}$ ) [10].

In the present study, we clarified that a larger amount of alcohol consumption was a risk factor for the incidence of proteinuria and CKD in women. The findings of the present study, as well as a previous study [15], suggest that avoiding excess alcohol consumption might be an important lifestyle modification to prevent kidney dysfunction, especially in women.

Although the precise mechanism of the nephrotoxic effect of alcohol is unsolved [26], one of the plausible mechanisms by which excess alcohol consumption induces kidney dysfunction is considered to be by inducing the depression of nephrin and podocin in podocytes, which causes proteinuria, leading to kidney dysfunction, and which is mediated by oxidative stress [27]. Furthermore, previous studies have shown that higher alcohol consumption is associated with an increased risk of future hypertension [28], leading to the incidence of proteinuria [22]. In the present study, the difference in blood pressure between each alcohol consumption group was not clinically significant, suggesting that the difference in blood pressure between each alcohol consumption group did not influence the occurrence of kidney dysfunction. Conversely, another study suggested that alcohol consumption might improve kidney antioxidant activities and capacity; namely, a small amount of ethanol pretreatment can increase the activities of inducible nitric oxide synthase and antioxidant capacities in the kidneys, which ameliorated oxidative stress in a bilateral renal ischemia reperfusion simulation model as a compensatory mechanism [29]. However, the optimal range of alcohol consumption to induce a positive effect on kidney function is unknown. Thus, further studies should evaluate this issue.

Regarding the mechanism for the sex difference in the impact of alcohol on kidney function, one possibility may be the different pharmacokinetics of alcohol between males and females. Compared with males, females are more likely to have higher concentrations of alcohol, partly because females, with a lower proportion of body water, have a smaller distribution volume of alcohol [15, 30]. Furthermore, it might be due to the differences in metabolism of alcohol because of lower activity of alcohol dehydrogenase in females than in males, leading to a higher concentration of alcohol in women than in men, even within a similar level of alcohol consumption [29]. Further studies should be conducted to explain these mechanisms.

This study has several limitations. First, self-reported alcohol consumption may be biased. Second, in the present study, proteinuria was measured using a dipstick, but dipstick tests are more likely to yield falsepositive and false-negative results than specific laboratory methods. Third, because of the retrospective nature of the present study, confounding factors such as excess alcohol consumption, unhealthy 
behaviors, and calorie-dense and hypersaline foods could not be evaluated [31]. This should be evaluated in future studies.

\section{Conclusion}

The present general population-based retrospective cohort study revealed that daily higher alcohol consumption was significantly associated with a higher incidence of proteinuria and an eGFR $<60$ $\mathrm{mL} / \mathrm{min} / 1.73 \mathrm{~m}^{2}$ with a $25 \%$ decline in eGFR among women. Based on our results, high alcohol consumption is a modifiable lifestyle-related factor to prevent CKD in women. Women might be prone to high alcohol consumption with kidney dysfunction.

\section{Abbreviations}

BMI, body mass index, CKD, chronic kidney disease, eGFR, estimated glomerular filtration rate, ESRD, endstage renal disease

\section{Declarations}

\section{Ethics approval and consent to participate}

The protocol of the present study was approved by the Daido Hospital and Aichi Medical University (no. 2020-8). An opt-out approach was adopted regarding informed patient consent, according to the Japanese Ethical Guidelines for Medical and Health Research involving human subjects.

\section{Consent for publication}

Not applicable.

\section{Availability of data and materials}

All data generated or analyzed during this study are included in this published article.

\section{Competing interests}

The authors declare that they have no competing interests.

\section{Funding}

No funding.

\section{Author contributions}

Conceptualization, T.A. and M.Y., methodology, M.Y., formal analysis, M.A. and Y.K., investigation, A.T., resources, A.T., data curation, A.T., writing - original draft preparation, A.T. and M.Y., writing - review and 
editing, Y.I., visualization, T.K., supervision, S.B. T.I. H.N. S.I. H.K. H.S. All authors have read and agreed to the published version of the manuscript.

\section{Acknowledgements}

The authors are grateful to all the medical staff who participated in this study.

\section{References}

1. Couser WG, Remuzzi G, Mendis S, Tonelli M. The contribution of chronic kidney disease to the global burden of major noncommunicable diseases. Kidney Int. 2011,80(12):1258-1270.

2. Room R, Babor T, Rehm J. Alcohol and public health. Lancet. 2005,365(9458):519-530.

3. Wood AM, Kaptoge S, Butterworth AS, Willeit P, Warnakula S, Bolton T, et al. Risk thresholds for alcohol consumption: combined analysis of individual-participant data for 599912 current drinkers in 83 prospective studies. Lancet. 2018,391(10129):1513-1523.

4. Gmel G, Gutjahr E, Rehm J. How stable is the risk curve between alcohol and all-cause mortality and what factors influence the shape? A precision-weighted hierarchical meta-analysis. Eur J Epidemiol. 2003,18(7):631-642.

5. Di Castelnuovo A, Costanzo S, Bagnardi V, Donati MB, lacoviello L, de Gaetano G. Alcohol dosing and total mortality in men and women: an updated meta-analysis of 34 prospective studies. Arch Intern Med. 2006,166(22):2437-2445.

6. Wang C, Xue H, Wang Q, Hao Y, Li D, Gu D, et al. Effect of drinking on all-cause mortality in women compared with men: a meta-analysis. J Womens Health (Larchmt). 2014,23(5):373-381.

7. Stockwell T, Zhao J, Panwar S, Roemer A, Naimi T, Chikritzhs T. Do "moderate" drinkers have reduced mortality risk? A systematic review and meta-analysis of alcohol consumption and all-cause mortality. J Stud Alcohol Drugs. 2016,77(2):185-198.

8. Schaeffner ES, Kurth T, de Jong PE, Glynn RJ, Buring JE, Gaziano JM. Alcohol consumption and the risk of renal dysfunction in apparently healthy men. Arch Intern Med. 2005,165(9):1048-1053.

9. White SL, Polkinghorne KR, Cass A, Shaw JE, Atkins RC, Chadban SJ. Alcohol consumption and 5year onset of chronic kidney disease: the AusDiab study. Nephrol Dial Transplant. 2009,24(8):24642472.

10. Koning SH, Gansevoort RT, Mukamal KJ, Rimm EB, Bakker SJ, Joosten MM, et al. Alcohol consumption is inversely associated with the risk of developing chronic kidney disease. Kidney Int. 2015,87(5):1009-1016.

11. Hsu YH, Pai HC, Chang YM, Liu WH, Hsu CC. Alcohol consumption is inversely associated with stage 3 chronic kidney disease in middle-aged Taiwanese men. BMC Nephrol. 2013,14:254.

12. Shankar A, Klein R, Klein BE. The association among smoking, heavy drinking, and chronic kidney disease. Am J Epidemiol. 2006,164(3):263-271. 
13. Buja A, Scafato E, Baggio B, Sergi G, Maggi S, Rausa G, et al. Renal impairment and moderate alcohol consumption in the elderly. Results from the Italian Longitudinal Study on Aging (ILSA). Public Health Nutr. 2011,14(11):1907-1918.

14. Cheungpasitporn W, Thongprayoon C, Kittanamongkolchai W, Brabec BA, O'Corragain OA, Edmonds PJ, et al. High alcohol consumption and the risk of renal damage: a systematic review and metaanalysis. QJM. 2015,108(7):539-548.

15. Kimura Y, Yamamoto R, Shinzawa M, Isaka Y, Iseki K, Yamagata K, et al. Alcohol consumption and incidence of proteinuria: a retrospective cohort study. Clin Exp Nephrol. 2018,22(5):1133-1142.

16. Sato KK, Hayashi T, Uehara S, Kinuhata S, Oue K, Endo G, et al. Drinking pattern and risk of chronic kidney disease: the kansai healthcare study. Am J Nephrol. 2014,40(6):516-522.

17. Fuselli S, De Felice M, Morlino R, Turrio-Baldassarri L. A three year study on 14 VOCs at one site in Rome: levels, seasonal variations, indoor/outdoor ratio and temporal trends. Int J Environ Res Public Health. 2010,7(10):3792-3803.

18. Menon V, Katz R, Mukamal K, Kestenbaum B, de Boer IH, Siscovick DS, et al. Alcohol consumption and kidney function decline in the elderly: alcohol and kidney disease. Nephrol Dial Transplant. 2010,25(10):3301-3307.

19. Reynolds K, Gu D, Chen J, Tang X, Yau CL, Yu L, et al. Alcohol consumption and the risk of end-stage renal disease among Chinese men. Kidney Int. 2008,73(7):870-876.

20. Park M, Lee SM, Yoon HJ. Association between alcohol intake and measures of incident CKD: an analysis of nationwide health screening data. PLoS One. 2019,14(9):e0222123.

21. Yamagata K, Ishida K, Sairenchi T, Takahashi H, Ohba S, Shiigai T, et al. Risk factors for chronic kidney disease in a community-based population: a 10-year follow-up study. Kidney Int. 2007,71(2):159-166.

22. Matsuo S, Imai E, Horio M, Yasuda Y, Tomita K, Nitta K, et al. Revised equations for estimated GFR from serum creatinine in Japan. Am J Kidney Dis. 2009,53(6):982-992.

23. Levin A, Stevens PE. Summary of KDIGO 2012 CKD guideline: behind the scenes, need for guidance, and a framework for moving forward. Kidney Int. 2014,85(1):49-61.

24. Kelly JT, Su G, Zhang L, Qin X, Marshall S, González-Ortiz A, et al. Modifiable lifestyle factors for primary prevention of CKD: a systematic review and meta-analysis. J Am Soc Nephrol. 2021,32(1):239-253.

25. Uehara S, Hayashi T, Kogawa Sato K, Kinuhata S, Shibata M, Oue K, et al. Relationship between alcohol drinking pattern and risk of proteinuria: the Kansai healthcare study. J Epidemiol. 2016,26(9):464-470.

26. Fan Z, Yun J, Yu S, Yang Q, Song L. Alcohol consumption can be a "double-edged sword" for chronic kidney disease patients. Med Sci Monit. 2019,25:7059-7072.

27. Samadi M, Shirpoor A, Afshari AT, Kheradmand F, Rasmi Y, Sadeghzadeh M. Chronic ethanol ingestion induces glomerular filtration barrier proteins genes expression alteration and increases matrix metalloproteinases activity in the kidney of rats. Interv Med Appl Sci. 2018,10(3):171-177. 
28. Yuan Q, Hong S, Han S, Zeng L, Liu F, Ding G, et al. Preconditioning with physiological levels of ethanol protect kidney against ischemia/reperfusion injury by modulating oxidative stress. PLoS One. 2011,6(10):e25811

29. Briasoulis A, Agarwal V, Messerli FH. Alcohol consumption and the risk of hypertension in men and women: a systematic review and meta-analysis. J Clin Hypertens (Greenwich). 2012,14(11):792-798.

30. Ammon E, Schäfer C, Hofmann U, Klotz U. Disposition and first-pass metabolism of ethanol in humans: is it gastric or hepatic and does it depend on gender? Clin Pharmacol Ther. 1996,59(5):503513.

31. Bundy JD, Bazzano LA, Xie D, Cohan J, Dolata J, Fink JC, et al. Self-reported tobacco, alcohol, and illicit drug use and progression of chronic kidney disease. Clin J Am Soc Nephrol. 2018,13(7):9931001.

\section{Figures}

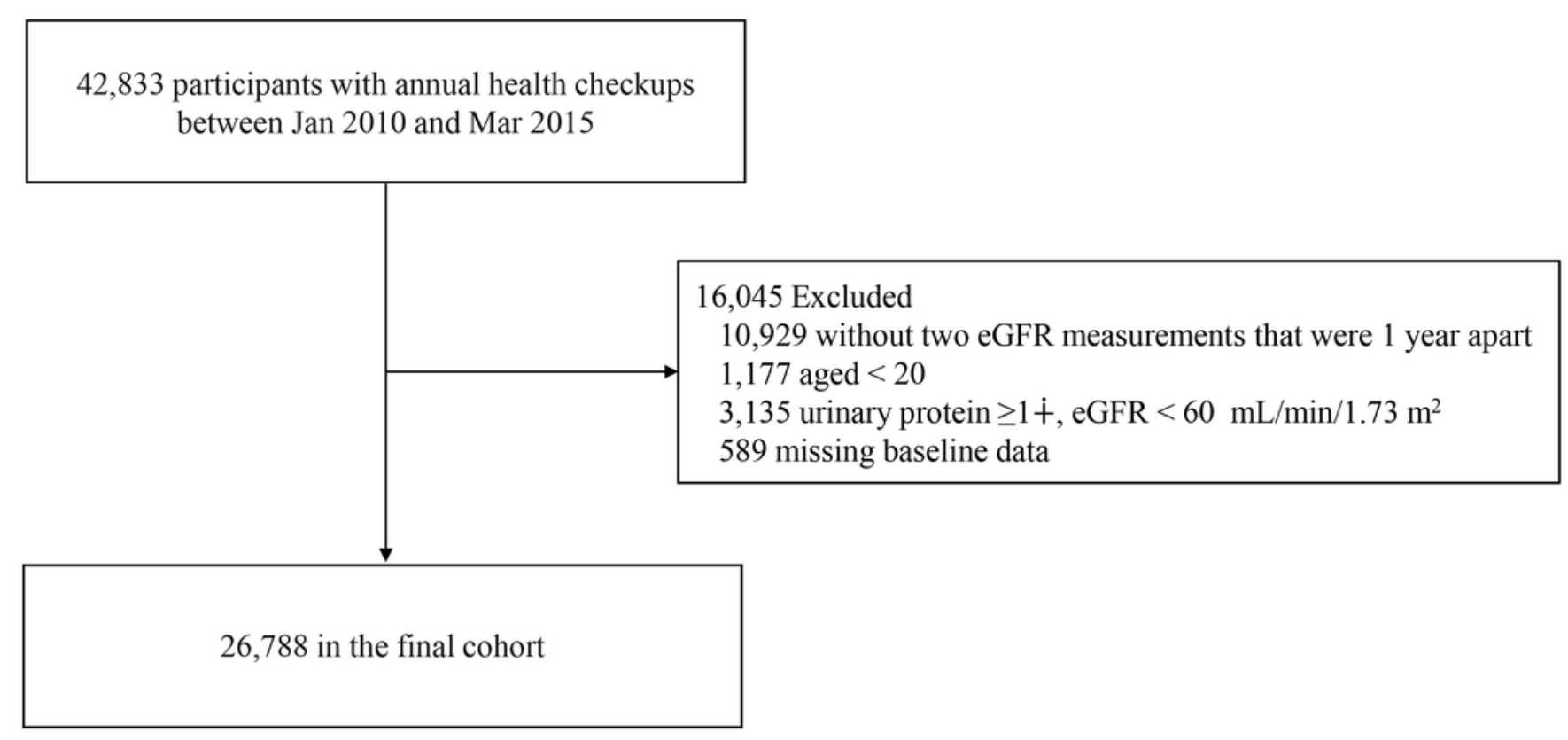

\section{Figure 1}

Flow diagram of the participants' selection. 

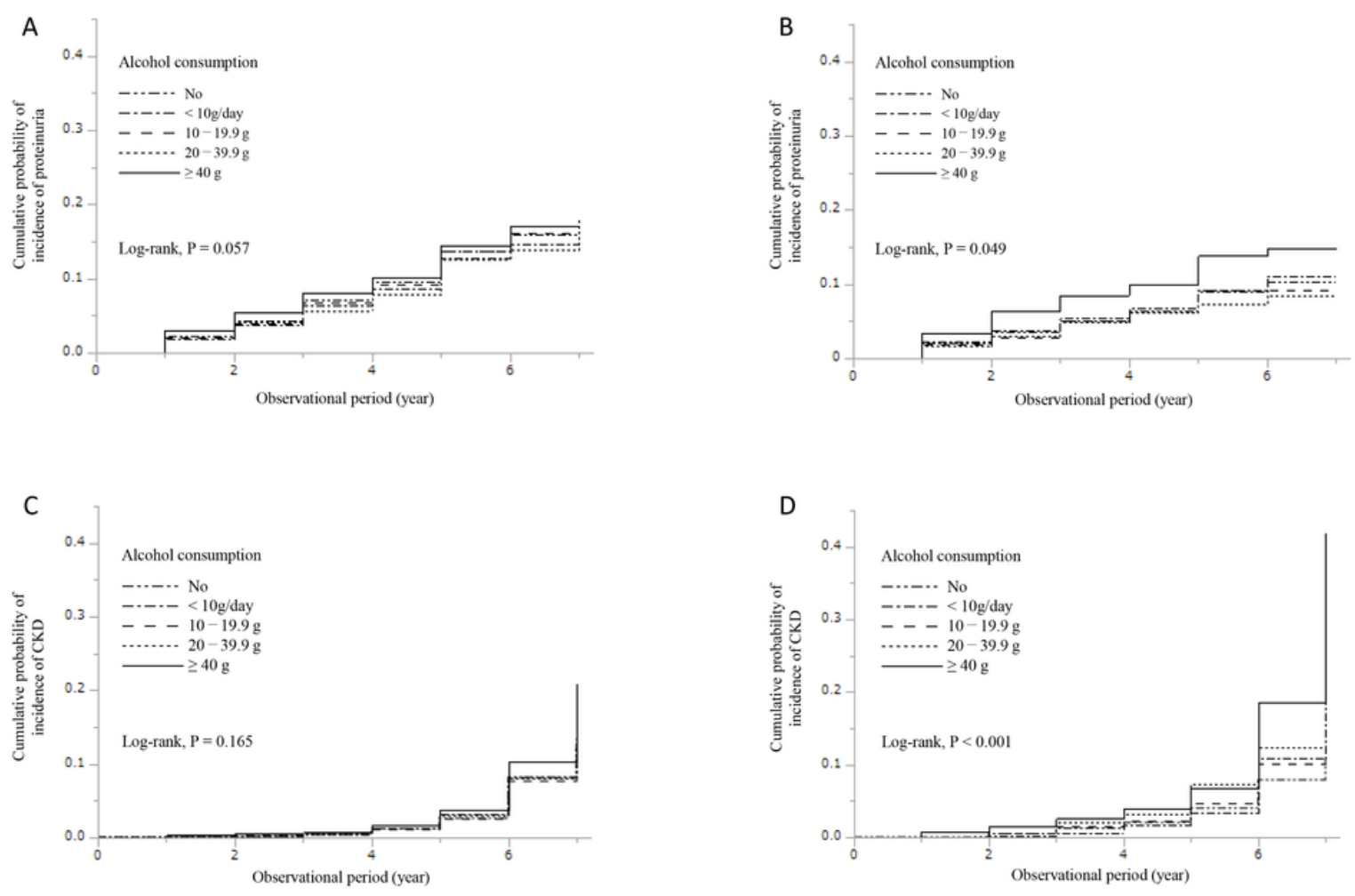

\section{Figure 2}

Cumulative probability based on alcohol consumption. The cumulative probability of incidence of proteinuria $(\geq 1+)$ in males $(A)$ and females $(B)$ and of CKD (eGFR $<60 \mathrm{~mL} / \mathrm{min} / 1.73 \mathrm{~m} 2$ and a $25 \%$ decrease) in males (C) and females (D). 